\title{
Sensitivity and Linearity of Superconducting Radio-Frequency Single-Electron Transistors: Effects of Quantum Charge Fluctuations
}

\author{
Madhu Thalakulam, ${ }^{1,2}$ Z. Ji, ${ }^{1}$ and A. J. Rimberg ${ }^{1,2,3}$ \\ ${ }^{1}$ Department of Physics and Astronomy, Rice University, Houston, TX 77005 USA \\ ${ }^{2}$ Rice Quantum Institute, Rice University, Houston, TX 77005 USA \\ ${ }^{3}$ Department of Electrical and Computer Engineering, Rice University, Houston, TX 77005 USA
}

\begin{abstract}
We have investigated the effects of quantum fluctuations of quasiparticles on the operation of superconducting radio-frequency single-electron transistors (RF-SETs) for large values of the quasiparticle cotunneling parameter $\alpha=8 E_{J} / E_{c}$, where $E_{J}$ and $E_{c}$ are the Josephson and charging energies. We find that for $\alpha>1$, subgap RF-SET operation is still feasible despite quantum fluctuations that renormalize the SET charging energy and wash out quasiparticle tunneling thresholds. Surprisingly, such RF-SETs show linearity and signal-to-noise ratio superior to those obtained when quantum fluctuations are weak, while still demonstrating excellent charge sensitivity.
\end{abstract}

PACS numbers: 73.23.Hk, 74.40.+k, 85.35.Gv

The radio-frequency single electron transistor (RFSET) is a highly sensitive, fast electrometer, and has been suggested as a potentially quantum-limited linear amplifier suitable for measurements of individual electronic charges [1, 2, 3, 4]. Recent investigations have addressed use of the RF-SET as an electrometer [4, [5, [6], a readout device for charge based qubits [7, 8, 9], and a sensor for real-time electron counting experiments [10]. Linearity is a fundamental assumption of theoretical discussions of the quantum limits of amplifiers [1, 11]. Nonetheless, there has been no detailed investigation of the range of linear response for the RF-SET.

Most theoretical studies of RF-SET performance focus on normal metal SETs, either in the sequential tunneling 1, 12, 13] or cotunneling regimes [2], while most experiments are performed using a superconducting SET (SSET) 6, 7, 8, 10]. Transport in the SSET can be divided into two regimes, depending on the relative sizes of the bias voltage $V_{\mathrm{dc}}$ and superconducting gap $\Delta$ : abovegap $\left(e V_{\mathrm{dc}}>4 \Delta\right)$, dominated by Coulomb blockade of quasiparticles, and subgap $\left(e V_{\mathrm{dc}}<4 \Delta\right)$, dominated by combinations of quasiparticle and resonant Cooper pair tunneling known as Josephson-quasiparticle (JQP) cycles 14, 15. While the best charge sensitivities are found for above-gap operation [6], the SSET backaction - the rate at which it dephases a measured system - is largest there [1, 3, 9]. Recent work has focused on subgap operation for which backaction is significantly reduced, and shot noise is non-Poissonian [3, 7, 16]. Theoretical studies of quantum fluctuations in the SSET have been limited to above-gap cotunneling of quasiparticles [17]. In this Letter we find that linearity and subgap quantum charge fluctuations in superconducting RF-SETs are intimately related: as quantum fluctuations strengthen, linearity and signal-to-noise ratio (SNR) improve, while charge sensitivity remains excellent.

Our SSETs consist of a small island connected to macroscopic leads via two $\mathrm{Al} / \mathrm{AlO}_{x} / \mathrm{Al}$ tunnel junctions $\mathrm{J} 1(2)$ with normal state resistances $R_{1(2)}$ as illustrated

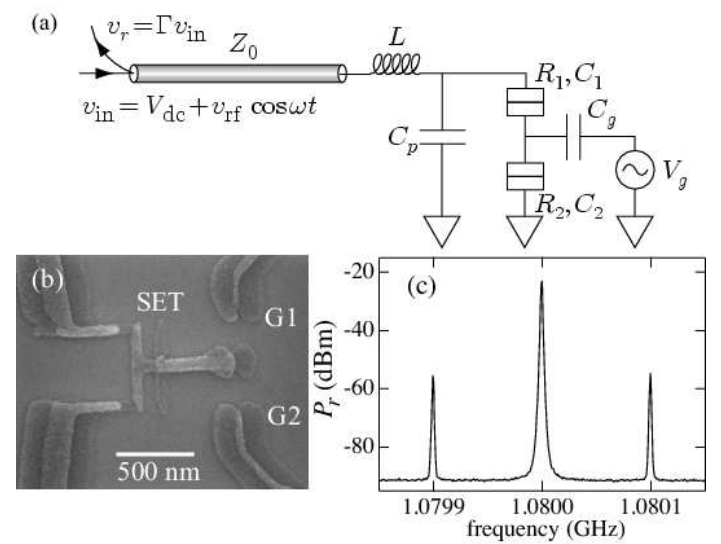

FIG. 1: (a) Schematic diagram of the SET illustrating RF operation. A voltage $v_{\text {in }}$ consisting of dc and $\mathrm{RF}$ biases $V_{\mathrm{dc}}$ and $v_{\text {rf }}$ is incident on a tank circuit consisting of an inductor $L$, a capacitor $C_{p}$, and the $\mathrm{SET}$, with tunnel junction resistances and capacitances $R_{1(2)}$ and $C_{1(2)}$. A small charge oscillation $q_{0} \cos \omega_{m} t$ modulates the reflection coefficient $\Gamma$ of the tank circuit and therefore the reflected voltage $v_{r}$. (b) Electron micrograph of S2 (taken after all measurements). Gates G1 and G2 were used vary the SET offset charge. (c) Power spectrum of $v_{r}$ for $q_{0}=0.063 e \mathrm{rms}$ and $\omega_{m} / 2 \pi=100 \mathrm{kHz}$. The measured sideband power and noise floor were used to find the charge sensitivity and SNR of the RF-SET.

in Fig. 1(a). We have fabricated and characterized three samples, $\mathrm{S} 1, \mathrm{~S} 2$, and $\mathrm{S} 3$ with total resistance $R_{n}=$ $R_{1}+R_{2}$ of 58,38 and $24 \mathrm{k} \Omega$; an electron micrograph of $\mathrm{S} 2$ is shown in Fig. 团(b). The samples were mounted on the mixing chamber of a dilution refrigerator at its base temperature of $20 \mathrm{mK}$. A Nb chip inductor $L \approx 120 \mathrm{nH}$ together with the parasitic capacitance $C_{p} \approx 0.2 \mathrm{pF}$ of the SET contacts constituted a tank circuit with resonant frequency $f_{L C} \approx 1 \mathrm{GHz}$ and quality factor $Q \approx 16$. We measured the samples' current-voltage $(I-V)$ characteristics in an asymmetric voltage-biased configuration [Fig. 1(a)] by varying the dc bias voltage $V_{\mathrm{dc}}$ in the ab- 
TABLE I: Sample parameters. Resistances are in $\mathrm{k} \Omega$, energies in $\mu \mathrm{eV}$, and areas in $10^{-3} \mu \mathrm{m}^{2}$.

\begin{tabular}{l|cccccccc}
\hline \hline & $R_{n}$ & $\Delta$ & $E_{c}$ & $E_{J}$ & $\alpha$ & $A_{\text {tot }}$ & $E_{c}^{0}$ & $E_{c}^{s}$ \\
\hline S1 & 58 & 200 & 230 & 22 & 0.78 & 4.1 & 254 & - \\
S2 & 38 & 200 & 250 & 34 & 1.08 & 3.4 & 291 & 258 \\
S3 & 24 & 190 & 162 & 54 & 2.65 & 5.0 & 218 & 162 \\
\hline \hline
\end{tabular}

sence of an RF excitation. Details of RF operation are similar to those discussed elsewhere [4, 6]. The SET offset charge $Q_{0}+q_{0} \cos \omega_{m} t$ consisted of a dc component $Q_{0}$ that set the overall working point and an ac component of amplitude $q_{0}$ that modulated the reflected voltage $v_{r}$. Power spectra of $v_{r}$ [Fig. 1(c)] were used to determine the charge sensitivity $\delta q$ and SNR.

In Fig. 2] we show representative $I-V$ characteristics of the samples in the superconducting state, measured for different $Q_{0}$, with $q_{0}=0$. For S1, we observe clear abovegap $\left(V_{\mathrm{dc}} \gtrsim 800 \mu \mathrm{V}\right)$ current modulation corresponding to Coulomb blockade of quasiparticle tunneling [Fig. 2(a)]. The main sub-gap features corresponding to the JQP [14, 15] cycles are sharp and clearly distinguished. As illustrated in Fig. 3, the simplest JQP cycle consists of resonant tunneling of a Cooper pair through one junction and dissipative tunneling of two quasiparticles through the other, transporting two electrons through the SET. The cycle can occur only when the transition $0 \rightarrow 1$ $(1 \rightarrow 0)$ is allowed, $i$. e., for $e V_{\mathrm{dc}}>E_{c}+2 \Delta$ where $E_{c}=e^{2} / 2 C_{\Sigma}$ is the charging energy of the SET and $C_{\Sigma}=C_{1}+C_{2}+2 C_{g}$ its total capacitance. While the JQP cycle is forbidden at lower bias, at $Q_{0} / e=n_{g} \approx \frac{1}{2}$ and $e V_{\mathrm{dc}}=2 E_{c}$ Cooper pair tunneling is resonant at both junctions and the double JQP (DJQP) cycle becomes possible. The fact that sequential tunneling cannot occur via either cycle for $2 E_{c} \lesssim e V_{\mathrm{dc}} \lesssim E_{c}+2 \Delta$ is reflected in $\mathrm{S} 1$ by a sharp drop in current at $V_{\mathrm{dc}} \approx 630 \mu \mathrm{V}$ just below the JQP feature.

As $R_{n}$ decreases, so does current modulation for $e V_{\mathrm{dc}}>4 \Delta$, consistent with suppression of the Coulomb blockade by quasiparticle cotunneling [17]: the modulation is reduced for S2, and nearly absent for S3 [Fig. 2](b) and (c)]. In contrast, features corresponding to the JQP cycles still exist but become progressively less sharp. Since these cycles involve both Cooper pair and quasiparticle tunneling, we hypothesize that subgap quantum fluctuations of quasiparticles are strong, while quantum fluctuations of Cooper pairs remain weak. Since to the best of our knowledge no theoretical description of subgap quantum charge fluctuations in the SSET exists, we provide simple arguments supporting our hypothesis.

We first compare with known results for above-gap transport. Following Ref. [17] we define a parameter $\alpha \equiv \frac{\Delta}{E_{c}} \frac{\pi \hbar}{e^{2}}\left(R_{1}^{-1}+R_{2}^{-1}\right)=\frac{8 E_{J}}{E_{c}}$ characterizing the strength of quantum fluctuations for quasiparticles, assuming $R_{1(2)}=R_{n} / 2$ and using the Ambegaokar-Baratoff rela-

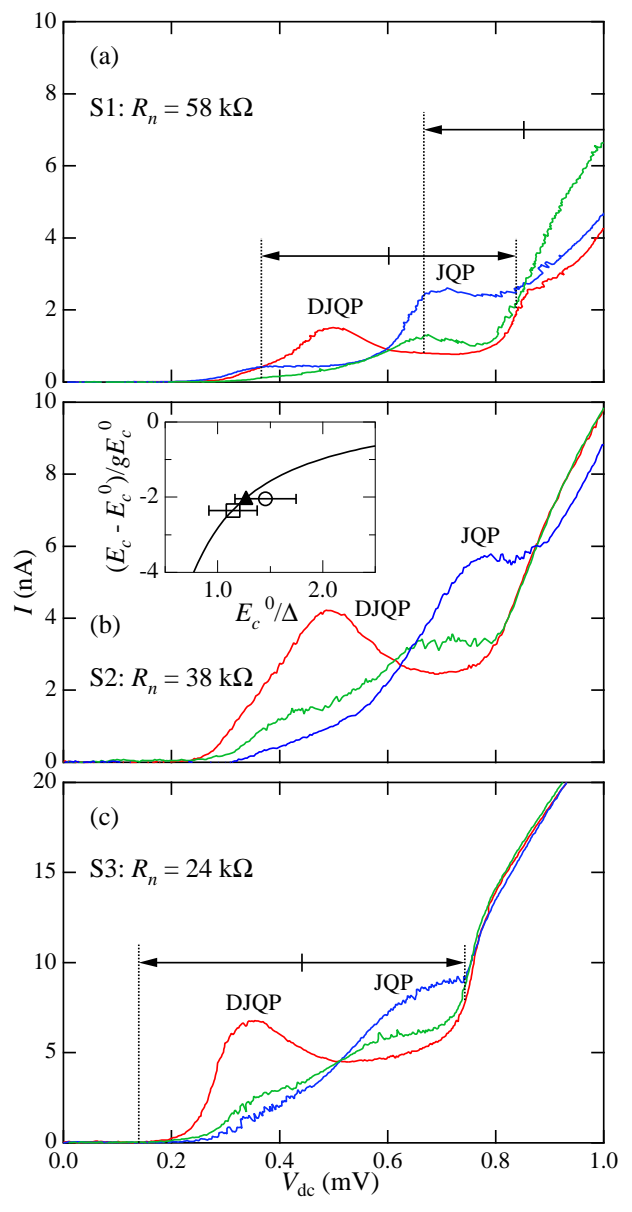

FIG. 2: $\quad I-V$ characteristics for (a) $\mathrm{S} 1$ (b) S2 and (c) S3 (note scale change), were chosen for $Q_{0}$ showing the DJQP process (red), the JQP process (blue), and an intermediate value of $Q_{0}$ (green). The arrows and vertical hash mark show the peak-to-peak RF amplitude $2 Q v_{\mathrm{rf}}$ and dc bias $V_{\mathrm{dc}}$ for optimal RF-SET operation. Inset: variation in the measured charging energy $E_{c}$ relative to the bare charging energy $E_{c}^{0}$ for S1 (solid triangle), S2 (circle) and S3 (square). Error bars indicate uncertainty in $E_{c}^{0}$. Solid line: theoretical prediction.

tion for the Josephson coupling energy $E_{J}=\frac{\Delta}{4} \frac{R_{K}}{R_{n}}$ where $R_{K}=\frac{h}{e^{2}}$. Quantum fluctuations are negligible for $\alpha \ll 1$. Determining $E_{c}$ from the location of the DJQP peak and $E_{J}$ from the total junction resistance we calculate $\alpha$ as in Table【 None of our samples satisfies $\alpha \ll 1$, although for S1 $(\alpha=0.78)$ some above-gap Coulomb modulation survives. The progressively weakening modulation for S2 $(\alpha=1.08)$ and S3 $(\alpha=2.65)$, is consistent with previous results [17].

Cotunneling as described in Ref. 17] occurs only for $V_{\mathrm{dc}}>4 \Delta / e$ : it results in two quasiparticle excitations and transfers a single electron through the SET. Other virtual processes, however, remain important for $V_{\mathrm{dc}}<4 \Delta / e$. For normal SETs, $E_{c}$ can be renormalized by quantum charge fluctuations: e. g., 
(a) JQP

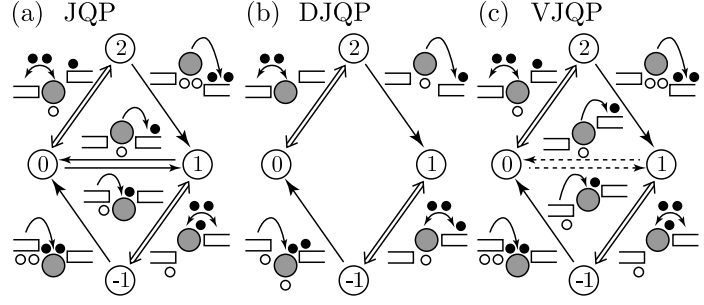

FIG. 3: Various JQP cycles. Here J2(1) is on the left (right) and $V_{\mathrm{dc}}>0$. Solid (empty) circles indicate quasielectrons (quasiholes) created during a cycle. (a) JQP cycle. Beginning in the state $n=0(n=1)$, where $\mathrm{n}$ is the number of excess electrons on the SET, the transition $0 \rightarrow 1(1 \rightarrow 0)$ is allowed, bringing Josephson tunneling through $\mathrm{J} 1(2)$ into resonance. Cooper pair tunneling $1 \Leftrightarrow-1(0 \Leftrightarrow 2)$ is interrupted by quasiparticle tunneling through the opposite junction $-1 \rightarrow$ $0(2 \rightarrow 1)$, completing the cycle. (b) DJQP cycle. When Josephson tunneling is simultaneously resonant through both $\mathrm{J} 1$ and $\mathrm{J} 2$, transport occurs via the sequence $0 \Leftrightarrow 2,2 \rightarrow 1$, $1 \Leftrightarrow-1,-1 \rightarrow 0$. (c) Proposed VJQP cycle. If the transition $0 \rightarrow 1(1 \rightarrow 0)$ is forbidden, it may still occur virtually. The remaining JQP transitions are allowed for relevant $V_{\mathrm{dc}}$.

near $n_{g}=0$, the effective charging energy $E_{c} \approx$ $E_{c}^{0}(1-4 g)$ where $g=R_{K} / \pi^{2} R_{n}$ is the dimensionless parallel conductance of the tunnel junctions and $E_{c}^{0}$ the bare charging energy; similar renormalization occurs in the superconducting state 5, 18]. Calculating the first-order energy shift due to transitions $n \rightarrow n \pm 1$, we find the renormalized charging en$\operatorname{ergy} E_{c}^{s}=E_{c}^{0}\left(1+g \frac{\Delta}{E_{c}^{0}} \Gamma\left[\frac{\Delta}{E_{c}^{0}}\left(1+2 n_{g}\right)\right]+\Gamma\left[\frac{\Delta}{E_{c}^{0}}\left(1-2 n_{g}\right)\right]\right)$ where $\Gamma(x)=\int_{0}^{\infty} K_{1}^{2}(u) e^{-x u} d u$ and $K_{1}(u)$ is a Bessel function.

Using the expression for $E_{c}^{s}$, we find empirically that $E_{c}^{0}=254 \mu \mathrm{eV}$ gives the measured $E_{c}$ for S1. We measure the total geometric junction area $A_{\text {tot }}$ for the samples with an estimated accuracy of $\pm 20 \%$, obtaining the values in Table \Setting $E_{c}^{0}=e^{2} / 2 C_{\Sigma}^{0}$ where $C_{\Sigma}^{0}=C_{1}^{0}+C_{2}^{0}+2 C_{g}$ and using $2 C_{g} \approx 80 \mathrm{aF}$, we obtain $C_{1}^{0}+C_{2}^{0}=195 \mathrm{aF}$ as the total unrenormalized junction capacitance for S1. Scaling this result according to $A_{\text {tot }}$ we find $C_{\Sigma}^{0}, E_{c}^{0}$ and finally $E_{c}^{s}$ for S2 and S3 [Table凹; agreement is excellent given the uncertainties in $A_{\text {tot }}$. The inset to Fig. 2 shows the relative difference between $E_{c}$ and $E_{c}^{0}$ scaled by $1 / g$. The results agree with theory to within our experimental accuracy, providing strong evidence that subgap quantum fluctuations of quasiparticles occur in our samples.

Virtual quasiparticle tunneling may also play a role in subgap transport, as suggested by the softening of the JQP cycle cutoff in S2 and S3. To illustrate such effects more clearly we show a plot of the $I\left(V_{\mathrm{dc}}, n_{g}\right)$ surface for S2 in Fig. प(a). The JQP resonances along the $0 \Leftrightarrow 2$ and $1 \Leftrightarrow-1$ lines and the DJQP peak at their intersection are clearly visible, but there is no sharp cutoff of the JQP process below the $1 \rightarrow 0(0 \rightarrow 1)$ thresholds.
For comparison, in Fig. 目(b) we show a simulation of the current in S2 based on sequential tunneling [19] at an elevated temperature and including photon-assisted tunneling due to an electromagnetic environment. Despite the extreme conditions the quasiparticle tunneling thresholds are clearly visible, and the SSET current drops nearly to zero between the JQP and DJQP features. The absence

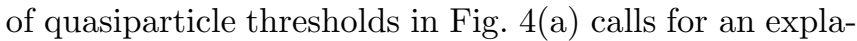
nation outside of the sequential tunneling picture.

A candidate process that could allow transport along the Cooper pair resonance lines between the JQP and DJQP features is illustrated schematically in Fig. 3(c). If below threshold the transition $1 \rightarrow 0(0 \rightarrow 1)$ occurs virtually, the transitions $0 \Leftrightarrow 2$ and $2 \rightarrow 1(-1 \Leftrightarrow 1$ and $1 \rightarrow 0$ ) are allowed, completing what we call the virtual JQP (VJQP) cycle. Two quasiparticle excitations are created, but two electrons are transferred through the SET, so that the process should be allowed for $e V>2 \Delta$. The energy barrier $E_{b}$ for $1 \rightarrow 0(0 \rightarrow 1)$ vanishes at threshold and climbs to $E_{b} \approx E_{c}+2 \Delta$ at the DJQP peak. The process can be neglected if the allowed quasiparticle tunneling rate $\Gamma_{\mathrm{qp}}$ is small compared to the inverse dwell time of the virtual quasiparticle: $\Gamma_{\mathrm{qp}} \ll E_{b} / \hbar$. Using $\Gamma_{\mathrm{qp}}=4 \Delta / e^{2} R_{n}$, this becomes $R_{n} \gg \frac{R_{K}}{\pi} \frac{2 \Delta}{E_{b}}$, which is violated for a range of voltages between the DJQP and JQP features. A detailed theoretical analysis is required to determine the contribution of the VJQP cycle to transport.

In contrast to the quasiparticle thresholds, features associated with Cooper pair tunneling are visible in both the data and the simulation, suggesting that the number of Cooper pairs is well defined. For the JQP process at resonance, the Cooper pair tunneling rate is $\Gamma_{\mathrm{cp}} \approx E_{J}^{2} / \hbar \Gamma_{\mathrm{qp}}=\frac{\pi}{8} \frac{E_{J}}{\hbar}[20]$. Demanding that energy broadening due to Cooper pair tunneling be small compared to the typical energy barrier $4 E_{c}$ for virtual tunneling gives $2 \hbar \Gamma_{\mathrm{cp}} / 4 E_{c}=\frac{\pi}{16} \frac{E_{J}}{E_{c}} \ll 1$, which is easily satisfied even for S3. For S2 and S3, then, quantum fluctuations are significant for quasiparticles but small for Cooper pairs.

We now turn to RF operation. Optimal operating conditions were selected as follows: a small charge oscillation $q_{0} \approx 0.006 e \mathrm{rms}$ was applied and the SNR determined from the power spectrum of $v_{r}$ as in Fig. 1(c). Subgap operation (all samples) and above-gap operation (S1) were optimized over dc bias $V_{\mathrm{dc}}$, rf bias $v_{\mathrm{rf}}$ and offset charge $Q_{0}$. We measured SNR versus input amplitude $q_{0}$ for each optimization and determined the charge sensitivity $\delta q$ using $\delta q=\frac{q_{0}}{\sqrt{\mathrm{BW}}} 10^{-\mathrm{SNR} / 20}$ where the resolution bandwidth $\mathrm{BW}=1 \mathrm{kHz}$ and $\mathrm{SNR}$ is in $\mathrm{dB}[\underline{6}]$.

The optimized biases for S1 and S3 are indicated in Fig. 2 and the results of the $\delta q$ and SNR measurements in Fig. [5. For S1 the best $\delta q=9 \times 10^{-6} e / \sqrt{\mathrm{Hz}}$ was found for $V_{\mathrm{dc}}=860 \mu \mathrm{V}$, consistent with previous results [6]. Linearity, however, was poor: as $q_{0}$ increases, the measured SNR rapidly becomes sublinear, and $\delta q$ 


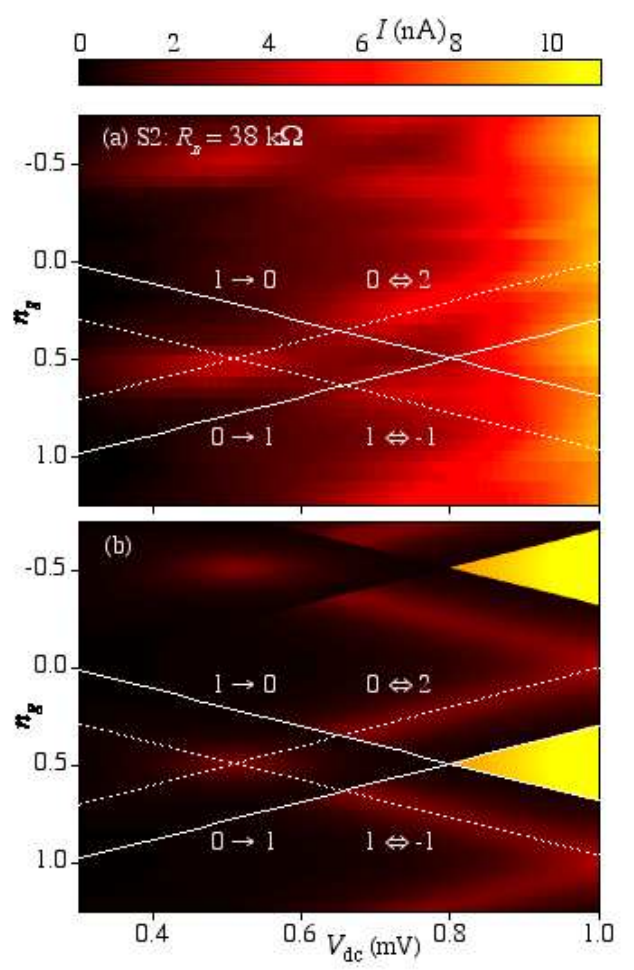

FIG. 4: False color images of $I\left(V_{\mathrm{dc}}, n_{g}\right)$ for (a) S2 at $T=20 \mathrm{mK}$ (b) a simulation at $T=200 \mathrm{mK}$ assuming an electromagnetic environment with impedance $R_{\mathrm{env}}=50 \Omega$ and temperature $T_{\text {env }}=1 \mathrm{~K}$. Cooper pair resonance lines $0 \Leftrightarrow 2(-1 \Leftrightarrow 1)$ and quasiparticle tunneling thresholds $1 \rightarrow 0$ $(0 \rightarrow 1)$ are indicated by the dashed and solid lines.

worsens [Fig. [5)]. Since $\delta q$ apparently does not saturate even for $q_{0}=4.5 \times 10^{-3} \mathrm{e} \mathrm{rms}$ it is unclear how small $q_{0}$ must be to achieve linear response. For sub-

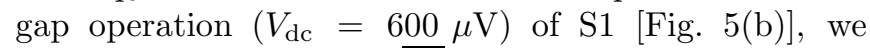
find $\delta q \approx 1.3 \times 10^{-5} e / \sqrt{\mathrm{Hz}}$, with SNR nearly linear to $q_{0} \lesssim 0.01 e \mathrm{rms}$. Since $\delta q$ appears close to saturation at $q_{0}=3.1 \times 10^{-3} e \mathrm{rms}$, we may have approached linear response.

For S3 the best operating point occurred at $V_{\mathrm{dc}}=$ $440 \mu \mathrm{V}$ [Fig. [5 (c)], between the DJQP and JPQ features with $\delta q \approx 1.2 \times 10^{-5} \mathrm{e} / \sqrt{\mathrm{Hz}}$, better than that for subgap operation of S1. Moreover, linearity was vastly improved: the SNR remains linear and $\delta q$ nearly flat to $q_{0}=0.038 \mathrm{e} \mathrm{rms}$ indicating that we have achieved linear response in this sample. For S2 (data not shown) the best $\delta q \approx 1.2 \times 10^{-5} \mathrm{e} / \sqrt{\mathrm{Hz}}$ also occurred subgap, and the SNR was linear to $q_{0} \approx 0.02 e \mathrm{rms}$.

We can now make some general statements about the effects of quantum fluctuations on RF-SET operation. For samples with smaller $\alpha$ such as $\mathrm{S} 1$, transport is fairly well described by the sequential tunneling picture: $I-V$ characteristics are sharp and vary strongly with $Q_{0}$ giving rise to excellent charge sensitivity. The same sharpness, however, prevents good linearity, since a large $q_{0}$

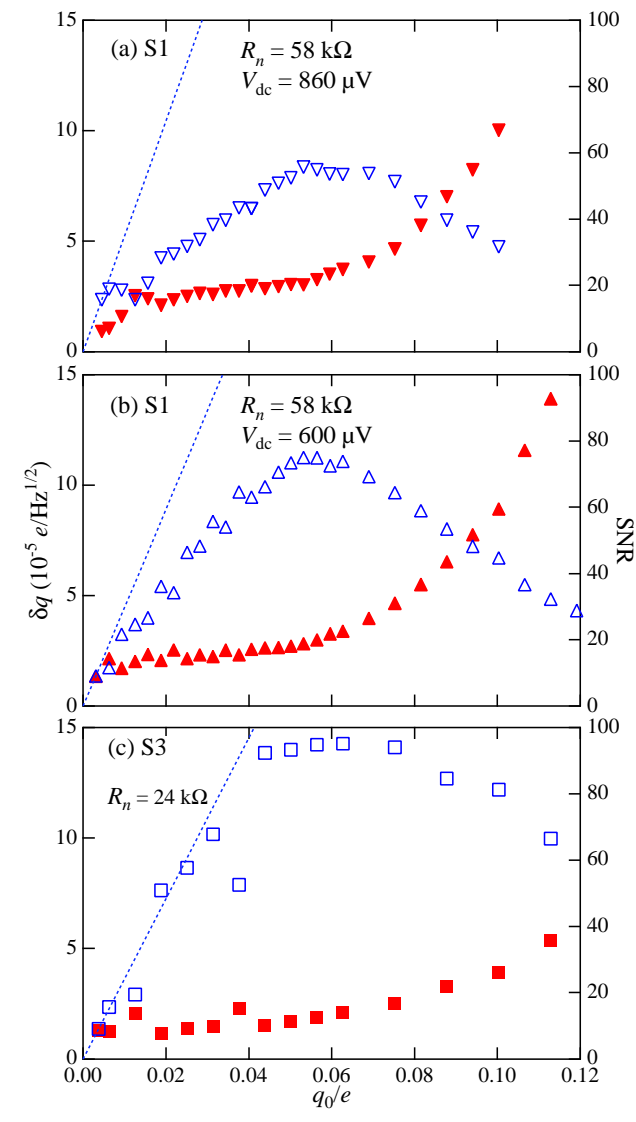

FIG. 5: Charge sensitivity $\delta q$ and SNR (linear scale) versus $q_{0}$ in $e$ rms for (a) S1, above gap, (b) S1, subgap, and (c) S3, subgap. Charge sensitivity (solid red symbols) is plotted on the left axis and SNR (open blue symbols) on the right. For reference, the SNR for linear response is plotted as the dashed lines for $\delta q$ measured at the smallest $q_{0}$.

necessarily moves the SET far from optimal operation. For samples with larger $\alpha$ such as S3, quantum fluctuations cause at least two important effects. First, the subgap features are smoothed and broadened, improving linearity: e. g., in S3 there is no "dead spot" between the DJQP and JQP features for which the SSET current is roughly independent of $Q_{0}$. Second, renormalization of $E_{c}$ moves the DJQP feature to lower bias, so that the optimal $\mathrm{rf}$ amplitude of about $\left(2 \Delta-E_{c}\right) / e$ increases with $\alpha$. Finally, the smaller $R_{n}$ simplifies impedance matching between the RF-SET and the $50 \Omega$ coaxial line.

In conclusion, we have investigated the influence of quantum charge fluctuations on the charge sensitivity and SNR of RF-SETs. We find that RF-SETs with $\alpha \gtrsim 1-2$ (strong quantum fluctuations) show both good linearity and good charge sensitivity. In contrast, RFSETs with $\alpha<1$ (weak quantum fluctuations) show poor linearity and only modestly better charge sensitivity. These findings assume particular importance given interest in the RF-SET as a potentially quantum-limited 
linear amplifier. We have achieved linear response only for subgap operation in samples with $\alpha \gtrsim 1$ for which quantum fluctuations of quasiparticles are substantial.

This work was supported by the National Science Foundation under Grant No. DMR-0242907, by the Army Research Office under Contact No. DAAD19-011-0616 and by the Robert A. Welch Foundation. We thank P. D. Dresselhaus and S. P. Benz for fabrication of the $\mathrm{Nb}$ chip inductors and S. M. Girvin for a critical reading of the manuscript.

[1] M. H. Devoret and R. J. Schoelkopf, Nature 406, 1039 (2000).

[2] A. Maassen van den Brink, Europhys. Lett. 58, 562 (2002).

[3] A. A. Clerk, S. M. Girvin, A. K. Nguyen, and A. D. Stone, Phys. Rev. Lett. 89, 176804 (2002).

[4] R. J. Schoelkopf, P. Wahlgren, A. A. Kozhevnikov, P. Delsing, and D. E. Prober, Science 280, 1238 (1998).

[5] K. W. Lehnert, B. A. Turek, K. Bladh, L. F. Spietz, D. Gunnarsson, P. Delsing, and R. J. Schoelkopf, Phys. Rev. Lett. 91, 106801 (2003).

[6] A. Aassimee, D. Gunnarsson, K. Bladh, P. Delsing, and R. Schoelkopf, Appl. Phys. Lett. 79, 4031 (2001).

[7] K. W. Lehnert, K. Bladh, L. F. Spietz, D. Gunnarsson,
D. I. Schuster, P. Delsing, and R. J. Schoelkopf, Phys. Rev. Lett. 90, 027002 (2003).

[8] A. Aassime, G. Johansson, G. Wendin, R. J. Schoelkopf, and P. Delsing, Phys. Rev. Lett. 86, 3376 (2001).

[9] Yu. Makhlin, G. Schön, and A. Shnirman, Rev. Mod. Phys. 73, 357 (2001).

[10] W. Lu, Z. Ji, L. Pfeiffer, K. W. West, and A. J. Rimberg, Nature 423, 422 (2003).

[11] C. M. Caves, Phys. Rev. D 26, 1817 (1982).

[12] A. N. Korotkov and M. A. Paalanen, Appl. Phys. Lett. 74, 4052 (1999).

[13] G. Johansson, A. Käck, and G. Wendin, Phys. Rev. Lett. 88, 046802 (2002).

[14] T. A. Fulton, P. L. Gammel, D. J. Bishop, L. N. Dunkleberger, and G. J. Dolan, Phys. Rev. Lett. 63, 1307 (1989).

[15] A. Maassen van den Brink, G. Schön, and L. J. Geerligs, Phys. Rev. Lett. 67, 3030 (1991).

[16] M. S. Choi, F. Plastina, and R. Fazio, Phys. Rev. Lett. 87, 116601 (2001).

[17] D. V. Averin, A. N. Korotkov, A. J. Manninen, and J. P. Pekola, Phys. Rev. Lett. 78, 4821 (1997).

[18] P. Joyez, V. Bouchiat, D. Esteve, C. Urbina, and M. H. Devoret, Phys. Rev. Lett. 79, 1349 (1997).

[19] W. Lu, K. D. Maranowski, and A. J. Rimberg, Phys. Rev. B 65, 060501 (2002).

[20] D. V. Averin and V. Ya. Aleshkin, Pis'ma Zh. Eksp. Teor. Fiz. 50, 331 (1989), [JETP Lett. 50, 367 (1989)]. 